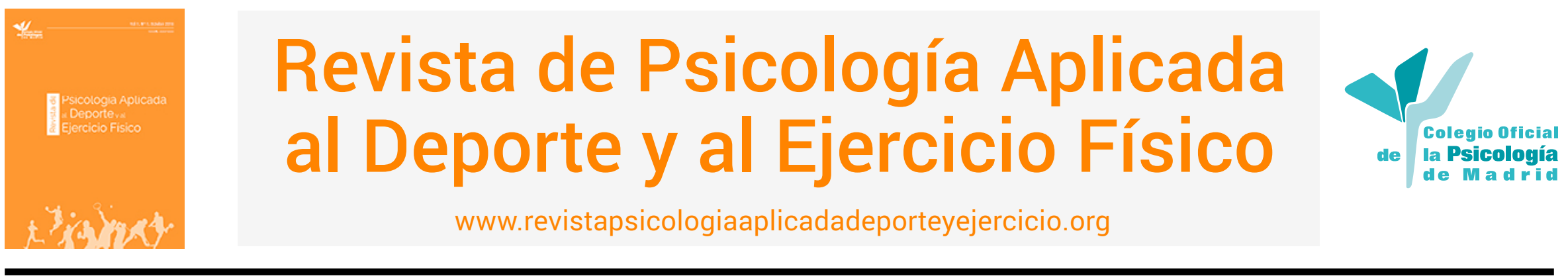

\title{
Relación entre resiliencia y rendimiento en deportistas. Revisión sistemática
}

\author{
Alicia María Sánchez Ortega \\ Universidad de Murcia, España \\ Francisco J. Ortín Montero \\ Universidad de Murcia, España
}

RESUMEN: La resiliencia es un constructo psicológico que, a pesar de no tener una única definición en el ámbito de la Psicología, podría definirse como la capacidad que tienen las personas para sobreponerse a las situaciones adversas y salir reforzadas de ellas. Este ha ganado mucha importancia en el contexto deportivo, en los últimos años. Esta revisión sistemática tiene como objetivo revisar la evidencia empírica existente, sobre dicho constructo y su relación con el rendimiento de los deportistas, teniendo en cuenta diferentes factores, intrapersonales e interpersonales. Para ello, siguiendo el proceso PRISMA se realizó una búsqueda sistemática en las bases de datos EBSCOhost, Pubmed y Dialnet, seleccionándose finalmente 22 estudios con unos criterios de inclusión y exclusión previamente establecidos como son: que los estudios fuesen artículos científicos (excluyendo revisiones, tesis...) publicados entre 2018 y 2020 con acceso abierto, que el idioma fuese español o inglés y, por otro lado, que las muestras fuesen de deportistas. Entre los resultados obtenidos destacan que existe un mayor nivel de resiliencia en deportistas que en no deportistas, también se puede observar una relación negativa entre este y el nivel de estrés o la ansiedad y lesiones deportivas y una relación positiva con el nivel de compromiso, además de la existencia de características personales (motivación intrínseca, tolerancia a la frustración, la autoeficacia) que los hacen más vulnerables al desarrollo y/o aumento de este. Asimismo, cabe destacar la influencia del entrenador y el entorno donde se desenvuelve, tanto para su desarrollo personal y deportivo, como el aumento del nivel de resiliencia. Finalmente, se concluye que la actividad deportiva desarrolla factores protectores en los deportistas, dando lugar a la resiliencia, la cual les permite superar las situaciones difíciles (presión, lesiones, derrotas), tanto dentro como fuera del ámbito deportivo, al mismo tiempo que disminuye la ansiedad y el estrés.

PALABRAS CLAVES: resiliencia, deporte y deportista

\section{Relationship between resilience and performance in athletes. Systematic review}

ABSTRACT: The term resilience is considered to be a psychological concept, which, although it doesn't have a specific definition in the field of psychology, could be defined as the ability that some people have to overcome unfavorable situations and come out stronger. A lot of importance has been given to this term in the last few years. This systematic review aims to reviewing the current empirical evidence which focused on the sport psychology and its relationship with athletes' efficiency. Different intrapersonal and interpersonal aspects were taken into consideration. To carry out this review, deep research was done by using the EBSCOhost, PubMed and Dialnet data and choosing 22 studies at last, with previously established inclusion and exclusion criteria such as: that the studies were scientific articles (excluding reviews, thesis...) published between 2018 and 2020 with unrestricted access, that the language was Spanish or English, and on the other hand, that the samples were athletes. Among the results obtained, it was striking that the sportspeople had a higher level of resilience than non-athletes, you can also observe the negative relationship between this construct and the level of stress or anxiety and sports injuries, and positive relationship with level of commitment, in addition to the existence of personal characteristics (intrinsic motivation, frustration tolerance, self-efficacy) that make them more vulnerable to its appearance and exacerbation. Moreover,

\footnotetext{
Alicia María Sánchez Ortega es graduada en Psicología

Francisco José Ortín Montero Doctor en Psicología

La correspondencia de este artículo debe enviarse a: Alicia María Sánchez Ortega al email: aliciamaria. sanchezo@um.es
} 
the influence of the coach and the setting should be mentioned, both for their personal and athletic development and for increasing their resilience. Finally, it's concluded that sport fosters protective factors in athletes, giving rise to resilience, which allows them to overcome difficult situations (pressure, injuries, losses), both on and off the sport's field, while reducing anxiety and stress.

KEYWORDS: resilience, sport, and athlete

\section{Relação entre resiliência e rendimento em atletas. Revisão sistemática}

RESUMO: Resiliência é um constructo psicológico que, apesar de não ter uma definição única no campo da psicologia, pode ser definida como a capacidade que as pessoas possuem de superar situações adversas e delas emergir reforçadas. Isto tornou-se muito importante no contexto desportivo nos últimos anos. Esta revisão sistemática tem como objetivo rever as evidências empíricas existentes sobre esse constructo e sua relação com o rendimento dos atletas, levando em consideração diferentes fatores, intrapessoais e interpessoais. Para tal, foi realizada uma pesquisa sistemática nas bases de dados EBSCOhost, Pubmed e Dialnet, acabando por se selecionar 22 estudos com critérios de inclusão e exclusão previamente estabelecidos, nomeadamente: que os estudos fossem artigos científicos (excluindo revisões, teses...) publicados entre 2018 e 2020, com acesso aberto, que o idioma fosse espanhol ou inglês e que as amostras fossem de atletas. A partir dos resultados obtidos, destacou-se o facto de haver um maior nível de resiliência em atletas do que em não atletas. Pode também observar-se uma relação negativa entre este e o nível de stress ou ansiedade e as lesões desportivas, bem como uma relação positiva com o nível de comprometimento. Verificou-se também a existência de características pessoais (motivação intrínseca, tolerância à frustração, autoeficácia) que os tornam mais vulneráveis ao desenvolvimento e/ou aumento do mesmo. Da mesma forma, importa destacar a influência do treinador e do ambiente em que atua, tanto para o seu desenvolvimento pessoal e desportivo, como para o aumento do nível de resiliência. Por fim, conclui-se que a atividade desportiva desenvolve fatores protetores nos atletas, dando origem à resiliência, a qual Ihes permite superar situações difíceis (pressão, lesões, derrotas), tanto dentro como fora do âmbito desportivo, ao mesmo tempo que reduz a ansiedade e o stress.

PALAVRAS-CHAVE: resiliência, desporto e atleta

Artículo recibido: 07/09/2021 | Artículo aceptado: 23/11/2021

Debido a la gran variedad de artículos publicados y a la cantidad de información de la que se dispone, nacen las revisiones sistemáticas. Pretenden ser una herramienta que recopila información sobre una cuestión planteada para obtener conclusiones con una mayor evidencia y poder tomar decisiones más acertadas (Moreno et al., 2018). Así pues, las revisiones sistemáticas se consideran estudios integrativos, observacionales, retrospectivos, los cuales consisten en combinar varios estudios primarios que examinan la misma cuestión, por lo que este tipo de estudios se está empezando a aplicar en aspectos de diagnóstico, pronóstico y estudios observacionales (Beltrán, 2005). Por tanto, al estar formados por múltiples artículos y fuentes de información, se considera que su evidencia es muy alta (Moreno et al., 2018).

El ejercicio físico y el deporte diario favorece la salud física, previniendo numeras patologías como la obesidad, hipertensión, diabetes o incluso ciertos tipos de cáncer (Ramírez et al., 2020). Por otra parte, el ejercicio físico tiene otros beneficios que no son tan populares, como es el bienestar psicológico, debido a que actualmente, en la literatura científica, están muy consolidados los conocimientos de los efectos de la práctica deportiva sobre la salud física y no tanto sobre el bienestar psicológico (Isorna y Felpeto, 2014). Sin embargo, existen numerosas investigaciones que tratan de indagar dicha relación y donde encontramos resultados tales como que la actividad física mejora la salud subjetiva, el estado de ánimo, la emotividad, disminuye la ansiedad, el estrés, incrementa la autoestima, entre otras (Cortês et al., 2020).

Más concretamente, la perspectiva de la psicología que pretende estudiar los constructos que entran en juego en este bienestar psicológico es la psicología positiva, de la cual fue el mayor exponente Martin Seligman (Contreras y Esguerra, 2006). Fue definida como el estudio científico de las experiencias positivas, los rasgos individuales positivos, las instituciones que facilitan su desarrollo y los programas que ayudan a mejorar la calidad de vida de los individuos, mientras previene o reduce la incidencia de la psicopatología. De forma más concreta, el campo de la psicología positiva se focaliza, a nivel subjetivo, en el bienestar, contento y satisfacción, esperanza y optimismo y por el flujo y felicidad (Contreras y Esguerra, 2006).

Hoy en día se considera que el éxito deportivo no sólo se alcanza con aptitudes físicas, sino que los factores psicológicos juegan un papel muy importante en el rendimiento. La 
psicología del deporte ha tratado de estudiar dichos factores que afectan directamente a los deportistas (Onturk, 2020).

A lo largo de la carrera de un deportista, este hace frente a diversos obstáculos e inconvenientes, donde se experimenta presión y estrés, tanto a nivel físico como mental, generando malestar emocional y estado de ánimo que influye en su desarrollo profesional y personal (Reche et al., 2020). Dicho estrés, puede deberse a derrotas, lesiones, presiones y expectativas, enfermedades, competitividad o conflictos con miembros del equipo y aparece por la interpretación de que las demandas del medio exceden los recursos de los que se dispone para hacer frente a estas, es decir, cuando consideran que sus recursos son escasos o insuficientes (Reche et al., 2020).. No obstante, a pesar de las dificultades que se puedan presentar, cabe destacar que los deportistas salen beneficiados al enfrentarse a estas, ya que las estrategias de afrontamiento que desarrollen podrán extrapolarlo a su vida cotidiana (Bicalho et al., 2020).

Teniendo en cuenta, que durante la práctica deportiva no siempre es posible sentir una experiencia positiva inmediata y que el deporte requiere la capacidad de persistir y adaptarse positivamente a eventos negativos, los investigadores argumentan que para obtener experiencias más positivas, mejores resultados y rendimiento, los deportistas necesitan desarrollar resiliencia (Pedro y Veloso, 2018).

La resiliencia, asociada con otros conceptos como la fortaleza mental, no implica tanto la invulnerabilidad al estrés, sino la habilidad de recuperarse de eventos negativos o estresantes (Giles et al., 2018). La resiliencia, en el área de la psicología, no tiene una única definición, sin embargo, se podrían aclarar dos componentes comunes en todas sus definiciones: a) la facultad de protección de la integridad propia bajo presión y 2) la facultad de proyectar un comportamiento positivo pese a las situaciones difíciles (Giles et al., 2018). Por otro lado, parece haber acuerdo entre autores de que la resiliencia se considera un proceso y no un estado y que se desarrolla en interacción del sujeto con el entorno (a nivel personal, familiar y comunitario), tanto con factores de riesgo (vivenciar momentos difíciles), factores protectores (red de apoyo, calidad de los vínculos), como características de personalidad (González et al., 2019).

Hay determinados factores que promueven la resiliencia como el 'locus de control interno' y el 'estilo atribucional; esto explica que las personas resilientes tengan una serie de características como: una mayor autoestima, autoeficacia, sentimientos de propia valía, seguridad de afrontar las situaciones de adversidad y un punto de vista de que los errores son consecuencia de factores que se pueden modificar (Cortês et al., 2020). Otra característica que suele estar presente en deportistas resilientes es una adecuada capa- cidad de afrontamiento, haciendo referencia a aquellas respuestas empleadas después de la evaluación de un evento estresante y aunque parezca sinónimo de resiliencia, se diferencia en que esta última influye en cómo se valora el evento (Kegelaers y Wylleman, 2019).

Teniendo todo esto en cuenta, se consideraría la actividad física deportiva como un potenciador de los factores protectores, incluso ante un escenario muy desfavorecedor, ya que el deporte es considerado una vía para desarrollar aspectos físicos, psicológicos y sociales, al mismo tiempo que minimiza los factores de riesgo (Cortês et al., 2020). Como indican estos autores, las características inclusivas, cooperativas, los cambios conductuales positivos, el aumento del rendimiento escolar, ocupación del tiempo libre, ocio, mejora del rendimiento motor, que dan sensación de control, competencia y autoeficacia, autoconcepto y autoestima, son algunos de los principales beneficios de la práctica deportiva, que desarrollan a su vez la resiliencia.

\section{Teorías que sustentan la Resiliencia}

En la psicología del deporte hay dos teorías fundamentales que sostienen el constructo "Resiliencia": el modelo de la resiliencia de Galli y Vealey (2008) y la teoría psicológica de la resiliencia de Fletcher y Sarkar (2012). En cuanto a la primera, defiende que un aspecto central en el proceso de resiliencia de los deportistas es que perciban que han conseguido resultados positivos por haber hecho frente a la adversidad (Reche et al., 2020).

Por otro lado, la Teoría psicológica de la Resiliencia (Fletcher y Sarkar, 2012) defiende que el componente principal de la resiliencia es la evaluación positiva y la metacognición hacia los estresores, es decir, percibir los eventos estresantes como oportunidades para crecer y desarrollarse. El que los deportistas hagan este tipo de interpretaciones depende de una serie de factores psicológicos, que en un buen equilibrio, llevarán a un rendimiento óptimo, que son: a) una personalidad positiva (ser extrovertido, optimista, tener estabilidad emocional, alta disposición a vivir nuevas experiencias); b) motivación, c) confianza en sí mismos (factor importante que parece sostener la relación estrés-resiliencia-rendimiento); d) concentración (capacidad de mantener y cambiar el foco atencional dependiendo de las demandas requeridas); e) apoyo social percibido (juega un papel fundamental como factor de protección ante la adversidad, incluso los deportistas que más rinden perciben que tienen un apoyo social de calidad) (Fletcher y Sarkar, 2012).

Por otro lado, Fletcher y Fletcher (2005), en su metamodelo para el estrés, las emociones y el desempeño, propone que 
los estresores surgen del entorno en el que se desenvuelve el sujeto y que está mediado por procesos de percepción, evaluación y afrontamiento. Por tanto, basándonos en este modelo, la resiliencia influye en el estrés a diferentes niveles; la evaluación de los estresores, la metacognición en respuesta a las emociones y la selección de afrontamiento (Fletcher y Sarkar, 2012).

Existe, además, otra teoría en la que varios investigadores basan sus estudios sobre resiliencia, la teoría de la autodeterminación (TAD) (Deci y Ryan, 2008) (Trigueros, Aguilar-Parra, Cangas-Díaz et al., 2019). Esta teoría plantea que hay tres necesidades psicológicas básicas: autonomía (sentimiento de responsabilidad), competencia y parentesco/relación (necesidad de tener una relación satisfactoria con otras personas), propone que la satisfacción de estas necesidades psicológicas básicas da lugar a un estado de bienestar, al mismo tiempo que fortalece los recursos (flexibilidad mental, tenacidad, creatividad, tolerancia a la frustración...) relacionados con la resiliencia y provocan la motivación en los seres humanos (intrínseca y extrínseca), produciendo un comportamiento más eficiente (Trigueros, Aguilar-Parra, Cangas-Díaz et al., 2019).

El objetivo de esta revisión sistemática es conocer la relación que existe entre resiliencia y rendimiento en los deportistas, a partir de la revisión de evidencia científica de los últimos años, con la finalidad de conocer, más concretamente, si dicho constructo se ha vinculado con los procesos que llevan a los deportistas a superar obstáculos o inconvenientes.

\section{Método}

Para llevar a cabo este estudio se siguieron las directrices de la Preferred Reporting Items for Systematic Reviews and Meta-Analyses (PRISMA) (Moher et al., 2009).

\section{Criterios de selección}

Se estableció una serie de criterios de inclusión que fueron: 1) estudios presentes en las bases de datos seleccionadas, utilizando las palabras claves determinadas, 2) que estudie la resiliencia en los deportistas y su relación con el rendimiento, 3) que estuviese publicado en el rango temporal determinado, 4) que el idioma fuese español o inglés. 5) que tuviese acceso abierto al artículo (Free Full Text), 6) que fuesen artículos científicos y 7) que especificasen el cuestionario aplicado.

En cuanto a los criterios de exclusión fueron: 1) aquellos cuya muestra eran estudiantes y no deportistas, 2) artículos duplicados, 3) revisiones sistemáticas, tesis doctorales, libros y capítulos de libro.
Sin embargo, no se determinó como criterio de exclusión ni la edad de los deportistas, ni el género, ni el nivel de competición.

\section{Búsqueda y selección de estudios}

El primer paso para la selección de los estudios necesarios en la presente revisión ha sido elegir las bases de datos en las que se realizará la búsqueda, que fueron: PubMed, Dialnet y EBSCOhost. Dentro de esta última se incluyeron todas las bases de datos que aglutina (38 bases de datos).

Para la búsqueda de los artículos científicos, se utilizaron las siguientes palabras clave: Resilience/Resiliencia/Sport/ Deporte/Athlete/Deportista. Además, se filtró por "enlace a texto completo" o "Free Full Text" y acotados por fecha, desde 2018 hasta 2020, debido a que se buscaba realizar una revisión lo más reciente posible, para que se adecuará a la realidad actual de los deportistas.

Figura 1. Diagrama de flujo PRISMA mostrando la búsqueda bibliográfica en cada una de las etapas

Identificación de estudios a través de bases de datos

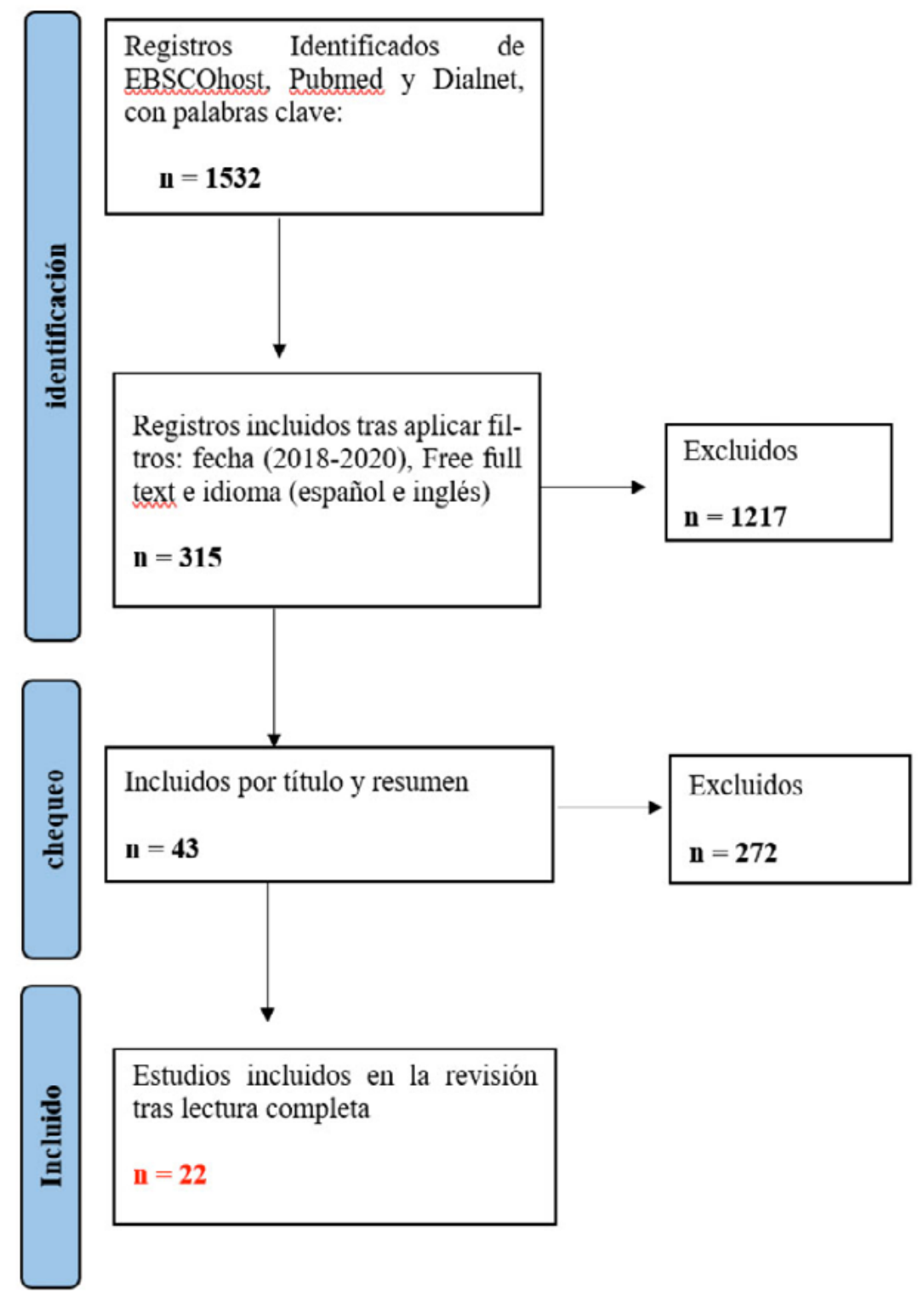

Revista de Psicología Aplicada al Deporte y al Ejercicio Físico (2021), 6, e16, 1-11 
El resultado de dicha búsqueda fue de 315 artículos, los cuales fueron leídos y analizados en base al título y resumen, excluyendo 272, según los criterios de exclusión nombrados anteriormente. Finalmente, fueron 43 artículos los que se seleccionaron para lectura completa y análisis. Tras dicha lectura, se excluyeron algunos artículos porque no aportaban datos compatibles con esta revisión, aunque también se eliminaron otros debido al difícil acceso que tenían.

Concluimos la búsqueda con 22 artículos. En la Figura 1, se presenta el diagrama de flujo donde aparece resumido el proceso de búsqueda.

\section{Resultados}

En la Tabla 1, se presenta el análisis y extracción de datos de los 22 artículos seleccionados. Esta tabla muestra, por un lado, los datos de cada estudio: título, autor o autores y año y, por otro lado, el tamaño de la muestra, el tipo de estudio que se ha llevado a cabo, la prueba o escala utilizada para la medida de la resiliencia, la fiabilidad de dicha prueba y, por último, los resultados más destacados del estudio relacionados con la resiliencia.

En primer lugar, cabe destacar que, la mayoría de los estudios se basan en un método descriptivo y transversal. Son descriptivos porque los objetivos de las investigaciones se basan en explicar y evaluar una situación y/o determinadas variables y sobre todo la posible la relación que exista entre estas. $Y$ son transversales ya que recogen los datos sobre dichas variables en un único momento.

A pesar de la gran variedad de pruebas utilizadas en los estudios analizados, las dos más destacadas son: La escala de Resiliencia de Connor-Davidson (CD-RISC) (Connor y Davidson, 2003) utilizada en un $13.6 \%$ de los artículos y la Escala de Resiliencia de Wagnild y Young (1993) en un 18.2\%.

En cuanto a la primera, debemos destacar que también encontramos su versión reducida CD-RISC10 (Connor y Davidson, 2003), o incluso su adaptación española de Notario et al. (2011).

Por otro lado, la Escala de Resiliencia de Wagnild y Young (1993), es considerada una escala válida y fiable, tanto para adolescentes como adultos. Sobre esta escala se han utilizado varias adaptaciones, entre ellas: una versión corta (RS13-A) aceptada y validada al portugués por Oliveira (2014).

No obstante, entre los artículos analizados también encontramos otras pruebas como: La adaptación de Vigário et al. (2009) de la Escala de Resiliencia en Contextos Deportivos (RSSC), Escala de Resiliencia en Contextos Deportivos (ERCD) (Trigueros et al., 2017), Cuestionario de Dureza men- tal Deportiva (SMTQ) (Sheard et al., 2009), Escala de Resistencia Mental (MTS) desarrollada por Madrigal et al. (2013), Escala de Resiliencia Psicológica Corta desarrollada por Smith et al. (2008).

Todas las pruebas muestran una buena consistencia interna, con una Alfa de Cronbach mayor de .7. Sin embargo, encontramos un estudio que no nos indica la consistencia interna de la escala utilizada, dicho estudio es el de Giles et al. (2018), lo que supone una limitación para este estudio. La prueba utilizada en el estudio de Onturk (2020), tiene un Alfa de Cronbach menor de .7, por tanto, podemos decir que el instrumento tiene una baja consistencia.

Todos los estudios utilizan una muestra grande con un rango de entre $N=134$ y $N=1725$, lo que le da fiabilidad y permite generalizar los resultados. Aunque hay varios cuya muestra consideraría una limitación debido a que no son lo suficientemente grande como para poder extrapolar los resultados a la población general, sin embargo, son seleccionados porque son estudios bien planteados y que aportan buenas conclusiones. Esto ocurre en un $22.7 \%$ de los estudios seleccionados y son: el estudio de Codonhato et al. (2018) que sólo tiene 8 atletas, el estudio de Giles et al. (2018) con 38 deportistas, Arnau et al. (2018) con 74 y los estudios de Aranzana et al. (2018) y Atkinson y Martin (2020), con muestras de 82 y 87 respectivamente.

Y en cuanto a la edad de las muestras, podríamos establecer un rango de edad entre 8 y 34 años. Excepto Atkinson y Martin (2020), que tiene participantes de hasta 61 años y León-Guereño et al. (2020), cuya edad media de la muestra es de 40.4 años.

Dentro de la Psicología del Deporte se ha considerado que el deporte potencia ciertas características personales y consigo la resiliencia. Siguiendo esta misma regla, se debería considerar que el deporte es promotor de la resiliencia y, por tanto, los deportistas tendrán mayor nivel de resiliencia que los no deportistas. Cortês et al. (2020), nos muestran resultados favorables hacia esto.

También hay autores que establecen una relación entre resiliencia y el nivel de competición de los deportistas. Leon-Guereño et al. (2020), Reche et al. (2020) y Kristjánsdóttir et al. (2019), afırman que esta relación es positiva, es decir, a mayor nivel de competición, más resiliente es el deportista.

Además, la resiliencia parece estar relacionada con muchas variables, como el nivel de motivación intrínseca, tolerancia a la frustración, la autoeficacia, el compromiso, perseverancia, es decir, existen ciertas características personales que pueden favorecer un perfil resiliente. Algunos de los estudios de esta revisión nos indican la relación que hay entre estas y el nivel de resiliencia. Por ejemplo, los estudios 
de Trigueros, Aguilar-Parra, Álvarez et al. (2019) Trigueros, Aguilar-Parra, Cangas-Díaz et al. (2019) y el estudio de Ramírez-Granizo et al. (2020), nos hablan de la relación positiva que existe entre los sujetos con motivación intrínseca y resiliencia. Por otro lado, Brace et al. (2020) nos muestra la fuerte relación que existe entre autoeficacia y fortaleza mental. Pedro y Veloso (2018) afirman que los sujetos con un mayor compromiso tienen más nivel de resiliencia, al igual que los sujetos con mayor perseverancia, según Giles et al. (2018).

Como se ha dicho anteriormente, la resiliencia permite a las personas adaptarse de manera eficiente tras situaciones difíciles, o estresantes. Por tanto, algunos estudios tratan de estudiar si esta relación es, como se espera, negativa. Los estudios de Codonhato et al. (2018), Moen et al. (2019), muestran que, a mayor nivel de resiliencia, menor es el estrés percibido por los deportistas. Al mismo tiempo que los estudios de González-Hernández et al. (2020), Trigueros et al. (2020) y Arnau et al. (2018), nos muestran una relación negativa entre el nivel de resiliencia del deportista y el nivel de ansiedad que presenta.

Otro resultado a destacar es la relación que parece existir entre el nivel de resiliencia y las lesiones en deportistas. En este sentido, Leon-Guereño et al. (2020), afirman una relación negativa entre resiliencia de los deportistas y las le- siones, de manera que, a mayor número de lesiones, menor nivel de resiliencia. En la misma línea, Moo y Salis (2019) indican que la ausencia de lesión es un factor importante para un adecuado estilo de afrontamiento.

También parece tener gran relevancia sobre las características del deportista el comportamiento del entrenador, es decir, cuando el entrenador opta por una actitud más prosocial (Trigueros et al., 2020) o por un apoyo a la autonomía (Pedro y Veloso, 2018), el deportista desarrolla un mayor nivel de resiliencia. Moen et al. (2019) afirman que una alianza eficaz entre el entrenador y el deportista es crucial para un adecuado crecimiento personal del deportista; así pues, los entrenadores tienen una gran repercusión en la capacidad de los deportistas para superar la adversidad y adaptarse. Además, esta repercusión no sólo será a nivel de bienestar psicológico y emocional, sino que también influye en el desarrollo de la autoestima y autoconfianza, según Trigueros et al. (2020).

Por último, hay que añadir, que hay estudios que encuentran diferencias significativas con respecto a la edad, lugar de residencia y el género, mientras que otros no encuentran dichas diferencias, por lo que estas relaciones no tienen gran consistencia.

Tabla 1. Resumen de los estudios seleccionados sobre resiliencia y deporte $(n=22)$

\begin{tabular}{|c|c|c|c|c|c|c|}
\hline Autor/es & Título & Muestra & $\begin{array}{l}\text { Tipos de } \\
\text { estudio }\end{array}$ & Evaluación resiliencia & Fiabilidad & Resultado más destacado \\
\hline $\begin{array}{l}\text { Aranzana et al. } \\
\text { (2018) }\end{array}$ & $\begin{array}{l}\text { Relación de la carga inter- } \\
\text { na de entrenamiento, op- } \\
\text { timismo y resiliencia con } \\
\text { los niveles de estrés-recu- } \\
\text { peración en nadadores }\end{array}$ & $\begin{array}{l}N=82 \\
\text { Edad: entre } 13 \\
\text { y } 29 \text { años }(M \\
=15,79, D T= \\
2,707)\end{array}$ & $\begin{array}{l}\text { Descriptivo/ } \\
\text { Longitudinal/ } \\
\text { Prospectivo }\end{array}$ & $\begin{array}{l}\text { Escala de Resiliencia de } \\
\text { Wagnild y Young, (1993) } \\
\text { en su versión española de } \\
\text { Ruiz, De la Vega, Poveda, } \\
\text { Rosado y Serpa, (2012) }\end{array}$ & .87 & $\begin{array}{l}\text { Ser nadador con un perfil resiliente alto se } \\
\text { asocia a una mayor reducción de la carga } \\
\text { interna de entrenamiento, al igual que ocu- } \\
\text { rre con los optimistas }\end{array}$ \\
\hline $\begin{array}{l}\text { Arnau et al. } \\
(2018)\end{array}$ & $\begin{array}{l}\text { Resiliencia y Ansiedad Pre- } \\
\text { competitiva en nadadores } \\
\text { en edad escolar. Un estudio } \\
\text { descriptivo }\end{array}$ & $\begin{array}{l}N=74 \\
\text { Edad: entre } 8 \\
\text { y } 14 \text { años }(M \\
=11,22 ; \quad S D= \\
1.67)\end{array}$ & $\begin{array}{l}\text { Descriptivo/ } \\
\text { Transversal }\end{array}$ & $\begin{array}{l}\text { CD-RISC en su adaptación } \\
\text { española de Notario et al., } \\
\text { (2011). }\end{array}$ & .85 & $\begin{array}{l}\text { Los resultados mostraron que aquellos na- } \\
\text { dadores con niveles muy bajos de resilien- } \\
\text { cia mostraron mayor ansiedad cognitiva } \\
\text { que sus compañeros con mayores niveles } \\
\text { de resiliencia }\end{array}$ \\
\hline $\begin{array}{l}\text { Codonhato et al. } \\
\text { (2018) }\end{array}$ & $\begin{array}{l}\text { Resilience, stress and in- } \\
\text { juries in the context of the } \\
\text { Brazilian elite rhythmic } \\
\text { gymnastics }\end{array}$ & $\begin{array}{ll}N=8 & \\
\text { Edad: } & \text { entre } \\
18 \text { y } 26 \text { años } \\
(20.4 \pm 2.5)\end{array}$ & $\begin{array}{l}\text { Descriptivo/ } \\
\text { Longitudinal/ } \\
\text { Prospectivo }\end{array}$ & $\begin{array}{l}\text { Escala de Resiliencia de } 10 \\
\text { ítems de Connor y Davidson } \\
\text { (CDRISC-10) }\end{array}$ & .72 & $\begin{array}{l}\text { La resiliencia se relaciona negativamente } \\
\text { con el estrés y este positivamente con las } \\
\text { lesiones }\end{array}$ \\
\hline $\begin{array}{l}\text { Giles et al. } \\
(2018)\end{array}$ & $\begin{array}{l}\text { Mental toughness and be- } \\
\text { havioural perseverance: A } \\
\text { conceptual replication and } \\
\text { extension }\end{array}$ & $\begin{array}{l}N=38 \\
\text { Edad media: } \\
21.18 \text { años }\end{array}$ & $\begin{array}{l}\text { Descriptivo/ } \\
\text { Transversal }\end{array}$ & $\begin{array}{l}\text { Cuestionario en línea (Qual- } \\
\text { trics LLC, Utah, EE. UU.) }\end{array}$ & & $\begin{array}{l}\text { la fortaleza mental es sobresaliente para } \\
\text { la perseverancia conductual en una tarea } \\
\text { física }\end{array}$ \\
\hline $\begin{array}{l}\text { Pedro y Veloso } \\
\text { (2018) }\end{array}$ & $\begin{array}{l}\text { Exploring resilience in } \\
\text { sports. Coach's autonomy } \\
\text { support and athletes en- } \\
\text { gagement -A contribute to } \\
\text { literatura }\end{array}$ & $\begin{array}{l}N=177 \\
\text { Edad: entre } 12 \text { y } \\
31 \text { años }\end{array}$ & $\begin{array}{l}\text { Descriptivo/ } \\
\text { Transversal }\end{array}$ & $\begin{array}{l}\text { Escala de Resiliencia de } \\
\text { Wagnild y Young (1993) y su } \\
\text { version reducida (RS13-A), } \\
\text { adaptada y validada al Por- } \\
\text { tugues por Oliveira }\end{array}$ & .82 & $\begin{array}{l}\text { Asociación positiva entre la resiliencia, el } \\
\text { apoyo a la autonomía de los entrenadores } \\
\text { y el compromiso de los atletas }\end{array}$ \\
\hline Dereceli (2019) & $\begin{array}{l}\text { An Examination of Concen- } \\
\text { tration and Mental Tough- } \\
\text { ness in Professional Bas- } \\
\text { ketball Players }\end{array}$ & $\begin{array}{l}N=290 \\
\text { Edad: entre } 16 \text { y } \\
26 \text { años }\end{array}$ & $\begin{array}{l}\text { Descriptivo/ } \\
\text { Transversal }\end{array}$ & $\begin{array}{l}\text { Cuestionario de Resistencia } \\
\text { Mental Deportiva (SMTQ- } \\
\text { 14) }\end{array}$ & $\begin{array}{l}.81 \text { para la con- } \\
\text { fianza; } .74 \text { para } \\
\text { continuidad; y } \\
.71 \text { para control }\end{array}$ & $\begin{array}{l}\text { La fortaleza mental y la confianza en ge- } \\
\text { neral diferían significativamente según la } \\
\text { edad del deporte. }\end{array}$ \\
\hline
\end{tabular}


Tabla 1. Resumen de los estudios seleccionados sobre resiliencia y deporte $(n=22)$ (Continuación)

\begin{tabular}{|c|c|c|c|c|c|c|}
\hline Autor/es & Título & Muestra & $\begin{array}{l}\text { Tipos de } \\
\text { estudio }\end{array}$ & Evaluación resiliencia & Fiabilidad & Resultado más destacado \\
\hline $\begin{array}{l}\text { González et al. } \\
\text { (2019) }\end{array}$ & $\begin{array}{l}\text { Exploring the Role of Resi- } \\
\text { lience and Basic Psycholo- } \\
\text { gical Needs as Antecedents } \\
\text { of Enjoyment and Boredom } \\
\text { in Female Sports }\end{array}$ & $\begin{array}{l}N=661 \\
\text { Edad media: } \\
14.74 \pm 3.91\end{array}$ & $\begin{array}{l}\text { Descriptivo/ } \\
\text { Transversal }\end{array}$ & $\begin{array}{l}\text { Escala de resiliencia de } \\
\text { Wagnild y Young (1993) y } \\
\text { adaptado por Ruiz et al. } \\
\text { (2012) y Suriá (2012) }\end{array}$ & .86 & $\begin{array}{l}\text { Cuando las deportistas son resilientes, se } \\
\text { favorece la satisfacción de sus necesidades } \\
\text { psicológicas y se dificulta su frustración }\end{array}$ \\
\hline $\begin{array}{l}\text { Kristjánsdóttir } \\
\text { et al. (2019) }\end{array}$ & $\begin{array}{l}\text { Psychological characte- } \\
\text { ristics in women football } \\
\text { players: Skills, mental tou- } \\
\text { ghness, and anxiety }\end{array}$ & $\begin{array}{l}N=142 \\
\text { Edad media: } \\
23.5 \text { años }\end{array}$ & $\begin{array}{l}\text { Descriptivo/ } \\
\text { Transversal }\end{array}$ & $\begin{array}{l}\text { El Cuestionario de Resis- } \\
\text { tencia Mental Deportiva } \\
\text { (SMTQ) de Sheard, Golby y } \\
\text { van Wersch, (2009) }\end{array}$ & .82 & $\begin{array}{l}\text { Los resultados parecen indicar que efecti- } \\
\text { vamente existe una relación entre MT y el } \\
\text { nivel del equipo }\end{array}$ \\
\hline $\begin{array}{l}\text { Moen et al. } \\
(2019)\end{array}$ & $\begin{array}{l}\text { Burnout and Perceived Per- } \\
\text { formance Among Junior } \\
\text { Athletes-Associations } \\
\text { with A_ective and Cognitive } \\
\text { Components of Stress }\end{array}$ & $N=670$ & $\begin{array}{l}\text { Descriptivo/ } \\
\text { Transversal }\end{array}$ & $\begin{array}{l}\text { La escala de resiliencia } \\
\text { para adultos (RSA) }\end{array}$ & Entre .63 y .81 & $\begin{array}{l}\text { El estudio actual indica que el agotamiento } \\
\text { del atleta y el rendimiento percibido pueden } \\
\text { explicarse por la resiliencia del atleta, que } \\
\text { tiene una relación negativa con el estrés } \\
\text { percibido }\end{array}$ \\
\hline $\begin{array}{l}\text { Moo y Solís } \\
(2019)\end{array}$ & $\begin{array}{l}\text { Resiliencia y lesiones en } \\
\text { deportistas universitarios }\end{array}$ & $\begin{array}{l}N=137 \\
\text { Edad: entre } 17 \\
\text { y } 26 \text { años, }(M \\
=20.65 \text { y } S D= \\
1.95)\end{array}$ & $\begin{array}{l}\text { Descriptivo/ } \\
\text { Transversal }\end{array}$ & $\begin{array}{l}\text { Cuestionario de Resiliencia } \\
\text { de Gonzales-Arratia, (2011). }\end{array}$ & $>.70$ & $\begin{array}{l}\text { La resiliencia de deportistas no lesionados } \\
\text { presentó una correlación negativa significa- } \\
\text { tiva la variable afrontamiento, lo que podría } \\
\text { indicar que la ausencia de lesión es un fac- } \\
\text { tor importante para un adecuado estilo de } \\
\text { afrontamiento }\end{array}$ \\
\hline $\begin{array}{l}\text { Reche et al. } \\
\text { (2019) }\end{array}$ & $\begin{array}{l}\text { Caracterización del depor- } \\
\text { tista resiliente y dependien- } \\
\text { te del ejercicio físico }\end{array}$ & $\begin{array}{l}N=387 \\
\text { Edad media: } \\
20.10(D T=4.2)\end{array}$ & $\begin{array}{l}\text { Descriptivo/ } \\
\text { Transversal }\end{array}$ & $\begin{array}{l}\text { Escala de Resiliencia de } \\
\text { Ruiz, De la Vega, Poveda, } \\
\text { Rosado y Serpa, (2012); } \\
\text { adaptación de Wagnild y } \\
\text { Young, (1993) al español }\end{array}$ & .89 & $\begin{array}{l}\text { Se observa que, en los atletas evaluados, } \\
\text { la élite presenta mayor resiliencia que la } \\
\text { no-élite. Se observa cómo la dependencia } \\
\text { al ejercicio físico aparece relacionada con } \\
\text { altos niveles de resiliencia }\end{array}$ \\
\hline $\begin{array}{l}\text { Trigueros, Agui- } \\
\text { lar-Parra, Álva- } \\
\text { rez et al. (2019) }\end{array}$ & $\begin{array}{l}\text { Emotion, Psychological We- } \\
\text { II-Being and Their Influence } \\
\text { on Resilience. A Study with } \\
\text { Semi-Professional Athletes }\end{array}$ & $\begin{array}{l}N=547 \\
\text { Edad: entre } 16 \\
\text { y } 19 \text { años }(M= \\
17.14)\end{array}$ & $\begin{array}{l}\text { Descriptivo/ } \\
\text { Transversal }\end{array}$ & $\begin{array}{l}\text { Escala de Resiliencia en } \\
\text { Contextos Deportivos } \\
\text { (RSSC) adaptado por Vi- } \\
\text { gário, serpa y Rosado }\end{array}$ & .84 & $\begin{array}{l}\text { La resiliencia se relaciona positivamente } \\
\text { con la motivación autónoma. }\end{array}$ \\
\hline $\begin{array}{l}\text { Trigueros, Agui- } \\
\text { lar-Parra, Can- } \\
\text { gas-Díaz et al. } \\
(2019)\end{array}$ & $\begin{array}{l}\text { The influence of the trai- } \\
\text { ner on the motivation and } \\
\text { resilience of sportspeople: } \\
\text { A study from the perspec- } \\
\text { tive of self-determination } \\
\text { theory }\end{array}$ & $\begin{array}{l}N=324 \\
\text { Edad: entre } 18 \\
\text { y } 34 \text { años }(M= \\
\text { 23.9; } S D=3.15)\end{array}$ & $\begin{array}{l}\text { Descriptivo/ } \\
\text { Transversal }\end{array}$ & $\begin{array}{lr}\text { Escala de } & \text { Resiliencia en } \\
\text { Contextos } & \text { Deportivos } \\
\text { (ERCD) } & \end{array}$ & .90 & $\begin{array}{l}\text { La resiliencia se relaciona positivamente } \\
\text { con la motivación autónoma }\end{array}$ \\
\hline $\begin{array}{l}\text { Leon et al. } \\
(2020)\end{array}$ & $\begin{array}{l}\text { The relationship of recrea- } \\
\text { tional runners' motivation } \\
\text { and resilience levels to the } \\
\text { incidence of injury: A me- } \\
\text { diation model }\end{array}$ & $\begin{array}{l}N=1725 \\
\text { Edad media: } \\
40.40 \pm 9.39\end{array}$ & $\begin{array}{l}\text { Descriptivo/ } \\
\text { Transversal }\end{array}$ & $\begin{array}{l}\text { Escala de Resiliencia de } \\
\text { Connor y Davidson (CD- } \\
\text { RISC) }\end{array}$ & .87 & $\begin{array}{l}\text { La resiliencia se relaciones negativamente } \\
\text { con las lesiones. Cuanto mayor es el nivel } \\
\text { de competición, más resilientes son. }\end{array}$ \\
\hline $\begin{array}{l}\text { González et al. } \\
\text { (2020) }\end{array}$ & $\begin{array}{l}\text { Resilient Resources in You- } \\
\text { th Athletes and Their Re- } \\
\text { lationship with Anxiety in } \\
\text { Di_erent Team Sports }\end{array}$ & $\begin{array}{l}N=241 \\
\text { Edad media: } \\
15.36 \quad(S D= \\
1.07)\end{array}$ & $\begin{array}{l}\text { Descriptivo/ } \\
\text { Transversal }\end{array}$ & $\begin{array}{l}\text { Versión en Español de la Es- } \\
\text { cala de Resiliencia (RS-14) }\end{array}$ & .80 & $\begin{array}{l}\text { La resiliencia se relaciona negativamente } \\
\text { con la ansiedad }\end{array}$ \\
\hline $\begin{array}{l}\text { Cortês et al. } \\
(2020)\end{array}$ & $\begin{array}{l}\text { The Resilience of Adoles- } \\
\text { cent Participants in Social } \\
\text { Projects } \\
\text { for Sport }\end{array}$ & $\begin{array}{l}N=134 \\
\text { Edad: entre } 12 \text { y } \\
17 \text { años }\end{array}$ & $\begin{array}{l}\text { Descriptivo/ } \\
\text { Transversal }\end{array}$ & $\begin{array}{l}\text { Escala de Resiliencia, adap- } \\
\text { tado por Wagnild y Young } \\
\text { (1993) }\end{array}$ & $P<.05$ & $\begin{array}{l}\text { Mayor nivel de resiliencia en aquellas per- } \\
\text { sonas que participaron en proyectos depor- } \\
\text { tivos que los que no }\end{array}$ \\
\hline $\begin{array}{l}\text { Ramírez et al. } \\
\text { (2020) }\end{array}$ & $\begin{array}{l}\text { Multidimensional Self Con- } \\
\text { cept Depending on Levels } \\
\text { of Resilience and the Mo- } \\
\text { tivational Climate Directed } \\
\text { towards Sport in School- } \\
\text { children }\end{array}$ & $\begin{array}{l}N=203 \\
\text { Edad: entre } 11 \\
\text { y } 13 \text { años }(M= \\
11.54)\end{array}$ & $\begin{array}{l}\text { Descriptivo } \\
\text { Transversal }\end{array}$ & $\begin{array}{l}\text { Escala de Resiliencia de } \\
\text { Connor y Davidson (CD- } \\
\text { RISC) }\end{array}$ & .86 & $\begin{array}{l}\text { A mayor motivación intrínseca basada en el } \\
\text { aprendizaje y la cooperación, mayores son } \\
\text { los valores de resiliencia en los niños }\end{array}$ \\
\hline
\end{tabular}


Tabla 1. Resumen de los estudios seleccionados sobre resiliencia y deporte $(n=22)$ (Continuación)

\begin{tabular}{|c|c|c|c|c|c|c|}
\hline Autor/es & Título & Muestra & $\begin{array}{l}\text { Tipos de } \\
\text { estudio }\end{array}$ & Evaluación resiliencia & Fiabilidad & Resultado más destacado \\
\hline $\begin{array}{l}\text { Brace et al. } \\
(2020)\end{array}$ & $\begin{array}{l}\text { Mental toughness and } \\
\text { self-efficacy of elite ul- } \\
\text { tra-marathon runners }\end{array}$ & $\begin{array}{l}N=140 \\
\text { Edad: menores } \\
\text { de } 18 \text { años }\end{array}$ & $\begin{array}{l}\text { Descriptivo/ } \\
\text { Transversal }\end{array}$ & $\begin{array}{l}\text { Cuestionario de dureza } \\
\text { mental deportiva (SMTQ). }\end{array}$ & $\begin{array}{l}\text { Confianza; } .80 \\
\text { constancia .74 } \\
\text { Control .7 }\end{array}$ & $\begin{array}{l}\text { la fortaleza mental y la autoeficacia se co- } \\
\text { rrelacionan fuertemente entre sí }\end{array}$ \\
\hline $\begin{array}{l}\text { Trigueros et al. } \\
(2020)\end{array}$ & $\begin{array}{l}\text { The Influence of the Trai- } \\
\text { ner's Social Behaviors on } \\
\text { the Resilience, Anxiety, } \\
\text { Stress, Depression and Ea- } \\
\text { ting Habits of Athletes }\end{array}$ & $\begin{array}{l}N=1547 \text { de- } \\
\text { portistas y } 127 \\
\text { entrenadores } \\
\text { Edad media: } \\
28.97 \text { años }\end{array}$ & $\begin{array}{l}\text { Descriptivo/ } \\
\text { Transversal }\end{array}$ & $\begin{array}{l}\text { Escala de Resiliencia de } \\
\text { Vigario, Serpa y Rosado's, } \\
\text { adaptada por Trigueros, } \\
\text { Aguilar, A Álvarez, Alcaraz } \\
\text { y Rosado }\end{array}$ & $>.70$ & $\begin{array}{l}\text { Los entrenadores con un comportamiento } \\
\text { prosocial se relacionaban positivamente } \\
\text { con el nivel de resiliencia del deportista. La } \\
\text { resiliencia está relacionada negativamente } \\
\text { con la ansiedad }\end{array}$ \\
\hline $\begin{array}{l}\text { Kilic y Yildirim } \\
(2020)\end{array}$ & $\begin{array}{l}\text { Investigation of Mental } \\
\text { Toughness Levels of Indi- } \\
\text { viduals Who Actively Do } \\
\text { Sports: A Sample of the } \\
\text { City of Elazig }\end{array}$ & $\begin{array}{l}N=156 \\
\text { Edad: entre } 16 \text { y } \\
31 \text { años }\end{array}$ & $\begin{array}{l}\text { Descriptivo/ } \\
\text { Transversal }\end{array}$ & $\begin{array}{l}\text { Escala de Resistencia Men- } \\
\text { tal (MTS), desarrollada por } \\
\text { Madrigal et al. y adaptado } \\
\text { al turco por Nevzat Erdogan }\end{array}$ & .86 & $\begin{array}{l}\text { Los deportistas masculinos tenían niveles } \\
\text { más altos de fortaleza mental en compara- } \\
\text { ción con las deportistas femeninas según } \\
\text { la variable de género. Además, no se obser- } \\
\text { varon diferencias significativas en cuanto a } \\
\text { las variables de estado civil, edad, nivel edu- } \\
\text { cativo, experiencia y rama deportivas }\end{array}$ \\
\hline $\begin{array}{l}\text { Atkinson y Mar- } \\
\text { tin (2020) }\end{array}$ & $\begin{array}{l}\text { Gritty, hardy, resilient, and } \\
\text { socially supported: A repli- } \\
\text { cation study }\end{array}$ & $\begin{array}{l}N=87 \\
\text { Edad: entre } 19 \text { y } \\
61 \text { años }\end{array}$ & $\begin{array}{l}\text { Descriptivo/ } \\
\text { Transversal }\end{array}$ & $\begin{array}{l}\text { Escalas de resiliencia de } \\
\text { Connor-Davidson de } 10 \\
\text { ítems, que fue una adapta- } \\
\text { ción del CD-RISC }\end{array}$ & .85 & $\begin{array}{l}\text { La resiliencia predijo el } 32 \% \text { de la varianza } \\
\text { en la satisfacción con la vida }\end{array}$ \\
\hline $\begin{array}{l}\text { Onturk et al. } \\
(2020)\end{array}$ & $\begin{array}{l}\text { Investigating the Psycholo- } \\
\text { gical Resilience of Students } \\
\text { in Sports Sciences Faculty }\end{array}$ & $\begin{array}{l}N=200 \\
\text { Edad: entre } 18 \text { y } \\
30 \text { años }\end{array}$ & $\begin{array}{l}\text { Descriptivo/ } \\
\text { Transversal }\end{array}$ & $\begin{array}{l}\text { Escala de Resiliencia Psi- } \\
\text { cológica Corta desarrollada } \\
\text { por Smith et al. (2008) }\end{array}$ & .66 & $\begin{array}{l}\text { El resultado del estudio reveló que la resi- } \\
\text { liencia psicológica se ve afectada por las } \\
\text { variables edad deportiva y lugar de residen- } \\
\text { cia }\end{array}$ \\
\hline
\end{tabular}

\section{Discusión}

La resiliencia es una variable psicológica, que ha tomado mucha importancia en la psicología del deporte, en los últimos años. Es definida como la capacidad de las personas para sobreponerse a las situaciones adversas y salir reforzadas de estas (García et al., 2014).

Así pues, el objetivo de esta revisión sistemática, como ya se ha explicado anteriormente, es conocer si existe una relación positiva entre el nivel de resiliencia de un deportista y su rendimiento y estudiar si favorece su afrontamiento ante las situaciones adversas, además de su tolerancia a la frustración, perseverancia, autoestima, etc. Para conocer si se cumple o no nuestra hipótesis, se hace una revisión sistemática a partir de estudios realizados con anterioridad, en lo que se investigue esta cuestión, entre 2018 y 2020 y localizados en bases de datos.

En primer lugar, cabe destacar que, debido a que el interés por la resiliencia es medianamente nuevo, los estudios realizados sobre este constructo son muy recientes y todavía, escasos, es decir, todavía queda mucho por indagar sobre este y su relación con el rendimiento de los deportistas.

La práctica deportiva, según Ramírez et al. (2020), es con- siderado un factor protector ante el estrés o la ansiedad, ya que permite el conocimiento intrapersonal del sujeto, descubriendo sus puntos débiles y sus puntos fuertes, lo que hace que mejore su autoconocimiento, autoestima y, por tanto, su autoconfianza y bienestar psicológico.

En cuanto factores individuales, parece ser que las personas más vulnerables a desarrollar resiliencia son aquellas con personalidad equilibrada y flexibles en cuanto a sus respuestas afectivas y fisiológicas, adaptándose a las circunstancias del ambiente (Reche et al., 2020).

También se considera un aspecto central en el desarrollo del deportista, la relación deportista-entrenador. Cuando el entrenador fomenta una relación basada en la autonomía del deportista, este pasa por un proceso de aprendizaje mucho más profundo y gratificante ante una situación adversa Trigueros, Aguilar-Parra, Cangas-Díaz et al. (2019). Además, es más probable que los deportistas evalúen positivamente los factores estresantes si su entrenador les proporciona los recursos adecuados y el apoyo social que necesitan (Moen et al., 2019), es decir, les ayuda a desarrollar ese nivel de resiliencia óptimo para su progreso. Así pues, es muy importante el papel del entrenador porque si aportan todo esto al deportista, puede dar lugar a un aumento de la autoestima, 
el autoconcepto y el bienestar; y consigo un perfil resiliente Trigueros, Aguilar-Parra, Álvarez et al. (2019). Por tanto, el entrenador debe tener en cuenta que no sólo debe formar a sus deportistas a nivel físico/técnico, sino también a nivel psicológico y sobre todo llevar mucho cuidado ya que una mala interacción entrenador-deportista puede provocar una pérdida de autoevaluación (Trigueros et al., 2020).

Todo deportista debe trabajar tanto los aspectos físicos y psicológicos, donde se potencie la resiliencia, entre otros que demande el deportista. La naturaleza de la resiliencia es considerada multidimensional, ya que implica factores individuales, familiares y del ambiente sociocultural donde se desenvuelve (Reche et al., 2020). Por tanto, para trabajar sobre la resiliencia, podría ser desde cualquiera de estas vías, sin embargo, los autores recomiendan que para potenciar la resiliencia es más efectivo trabajar sobre los factores protectores (autoeficacia, autoestima, apoyo social, optimismo), en lugar de reducir los factores de riesgo (ansiedad, estrés) (Aranzana et al., 2018).

También hay que tener en cuenta que para que el entrenamiento de resiliencia sea eficaz, debe enfocarse en tres aspectos: a) desarrollar cualidades/habilidades psicológicas protectoras, b) crear un entorno facilitador, equilibrando el desafío y el apoyo recibido y c) promover la mentalidad de superación, es decir, para conseguir una evaluación positiva de dichos desafíos, mediante sus pensamientos, emociones y recursos (Kegelaers y Wylleman, 2019).

Como alcance que pueden llegar a tener estos resultados en la práctica, pueden ser diversos. A nivel individual, puede resultar interesante que una persona que quiera reforzar su nivel de resiliencia, con el fin de disminuir su nivel de estrés y/o ansiedad a la hora de enfrentarse a ciertas situaciones de su vida, se inicie en la práctica deportiva, ya que como queda demostrado en los estudios realizados, el deporte te ayuda a desarrollarte como persona, aumentando el nivel de resiliencia, que es mayor en los deportistas que en los no deportistas.

A nivel de equipo, si la finalidad es potenciar la resiliencia del deportista para aumentar su rendimiento, sería conveniente trabajar tanto a nivel personal, desarrollando autoestima, la autoconfianza, el autoconocimiento, pero al mismo tiempo, trabajar con el entrenador, ya que la relación que se establece entre entrenador y deportista es determinante para el desarrollo de este último. Modificando o corrigiendo, si es preciso, la actitud del entrenador hacia el deportista, ya que esto haría que se aumentara la motivación intrínseca de este. Además de infundir el compromiso hacia lo que hace.

En cuanto a las limitaciones que puede tener esta revisión sistemática podría ser la falta de filtros, ya que haber filtrado por tamaño de la muestra y por coeficiente de fiabilidad, habría podido ser interesante, porque hay varios estudios cuyo tamaño de la muestra es pequeño y otros que no presentan el coeficiente de fiabilidad del test utilizado. Esto no invalida el estudio, pero si reduce su consistencia y fiabilidad.

Como propuestas para futuras investigaciones es estudiar si hay diferencias significativas en el nivel de resiliencia entre sujetos según la edad, el género y aspectos sociodemográficos, ya que hay un número muy reducido de estudios sobre esto y además se obtienen resultados contradictorios.

También podría ser interesante estudiar la relación que existe entre resiliencia y lesiones deportivas o número de lesiones, ya que los estudios sobre esto son insuficientes $y$, las lesiones deportivas son un aspecto muy estresante para estos y que puede marcar su carrera deportiva alterando su nivel de resiliencia.

Como última propuesta a posibles investigaciones futuras, sería estudiar, en deportes de equipo, si existen diferencias significativas entre el nivel de resiliencia y el papel que juega el deportista en el equipo, es decir, si hay diferencias entre titulares y suplentes, ya que esto también podría considerarse una situación de estrés, que requiere actitud de superación. Conocer si realmente existen diferencias podría ser muy útil en cuanto al trabajo psicológico con los deportistas que se pueda realizar.

\section{Conclusiones}

Para finalizar, según los resultados obtenidos sobre los estudios seleccionados, confirmamos la hipótesis planteada, llevando además a una serie de conclusiones.

La primera conclusión que destacar es que el deporte fortalece factores protectores del deportista a nivel intrapersonal, como interpersonal y son estos factores los que los hacen más proclives a potenciar la resiliencia. Esta es la razón por la que los deportistas presentan un mayor nivel de resiliencia que los no deportistas.

Además, es un factor que hace que disminuya el estrés y la ansiedad ante las situaciones adversas que pueden vivir los deportistas. Porque a mayor resiliencia en el deportista, menor va a ser el estrés percibido y la ansiedad generada, debido a su autopercepción y la valoración que hace de las demandas del medio.

También cabe destacar que los deportistas con mayor motivación intrínseca desarrollarán mayor resiliencia, ya que estos dedicarán todo su esfuerzo por placer y no por recompensa externa, así pues, será más fácil para estos afrontar y remontar ante dichas situaciones.

Como última conclusión, el entrenador y la actitud/posición que adopta tiene una gran influencia en el desarrollo de la resiliencia en deportistas. 


\section{Aplicaciones prácticas}

El conocimiento teórico riguroso de los conceptos en investigación tiene una repercusión que va más allá del propio trabajo publicado. En este sentido, para el establecimiento de programas específicos de entrenamiento psicológico, tanto en deportistas individuales como en equipos, resulta importante conocer las publicaciones existentes en relación a cada variable o constructo.

Las revisiones sistemáticas son herramientas metodológicas que acercan al profesional a aspectos esenciales de cara a una intervención más efectiva. En el caso de este artículo, hemos pretendido recabar la información científica sobre el constructo resiliencia y su posible relación con el rendimiento. Aunque es un término asociado en mayor medida a la salud, sabemos que una persona más saludable a través de una mayor resiliencia se enfrentará de forma más sana a las situaciones potencialmente estresantes y, por tanto, aumentará la probabilidad de mejorar su rendimiento. La actividad física y el deporte suponen contextos idóneos para el trabajo y desarrollo de la resiliencia, pues la persona se expone de forma continua a retos y dificultades que, si las supera de forma adecuada, aporta un aprendizaje y un granito de arena en su posible perfil resiliente. Por otro lado, la evaluación de la resiliencia en deportistas puede aportar información valiosa, junto con otras variables, de cara al trabajo de los técnicos que rodean al deportista, siempre asesorados por un psicólogo del deporte en la intervención específica.

\section{Referencias}

Aranzana, M., Salguero, A., Molinero, O., Boleto, A. F. y Márquez, S. (2018). Relación de la carga interna de entrenamiento, optimismo y resiliencia con los niveles de estrés-recuperación en nadadores. Cuadernos de Psicología del Deporte, 18(1), 43-54. https://doi.org/10.6018/cpd

Arnau, V. M., Checa, I. y Bohórquez, M. R. (2018). Resilience and Precompetitive Anxiety in swimmers of school age. A descriptive study. Información Psicológica, 175(1), 79-92. https://doi. org/10.14635/IPSIC.2018.115.

Atkinson, F. y Martin, J. (2020). Gritty, hardy, resilient, and socially supported: Areplication study. Disabilityand Health Journal, 13(1), Artículo 100839. https://doi.org/10.1016/j.dhjo.2019.100839

Beltrán, Ó. (2005). Revisiones sistemáticas de la literatura. Revista Colombiana de Gastroenterología, 20(1), 60-69.

Bicalho, C., Melo, G. y Noce, F. (2020). Resilience of athletes: a systematic review based on a citation network analysis. Cuadernos de Psicología del Deporte, 20(3), 26-40

Brace, A., George, K. y Lovell, G. (2020). Mental toughness and self-efficacy of elite ultra-marathon runners. PLOSONE, 15(11), Artículo e024128. https://doi.org/10.1371/journal.pone.0241284
Codonhato, R., Rubio, V., Pereira, P., Ferezin, C., Akawana, B., Pujals, C. y Fiorese, L. (2018). Resilience, stress and injuries in the context of the Brazilian elite rhythmic gymnastics. PLOS ONE, 13(12), Artículo e0210174. https://doi.org/10.1371/journal.pone.0210174

Contreras, F. y Esguerra, G. (2006). Psicología positiva: una nueva perspectiva en psicología. Diversitas, 2(2), 311. https://doi. org/10.15332/s1794-9998.2006.0002.10

Connor, K. M. y Davidson, J. R. (2003). Development of a new resilience scale: The Connor-Davidson Resiliencce Scale (CD-RISC). Depression and anxiety, 18(2), 76-82. https://doi. org/10.1002/da. 10113

Cortês, E., Dantas, M., Maia, R., Araújo, I. y Maia, E. (2020). The Resilience of Adolescent Participants in Social Projects for Sport. Ciência \& Saúde Coletiva, 25(3), 901-908. https://doi. org/10.1590/1413-81232020253.18362018

Deci E. y Ryan R. (2008). Self-Determination Theory: A macrotheory of human motivation, development, and health. Canadian Psychology, 49(3), 182-185.

Dereceli, A. (2018). An Examination of Concentration and Mental Toughness in Professional Basketball Players. Journal of Education and Training Studies, 7(1), 17-22. https://doi.org/10.11114/ jets.v7i1.3841

Fletcher, D. y Sarkar, M. (2012). Una teoría fundamentada de la resiliencia psicológica en campeones olímpicos. Psicología del deporte y el ejercicio, 13(5), 669-678. https://doi.org/10.1016/j. psychsport.2012.04.007

Galli, N. y Vealey, R. S. (2008). "Bouncing back" from adversity: Athletes' experiences of resilience. The Sport Psychologist, 22(3), 316-335. https://doi.org/10.1123/tsp.22.3.316

García, X., Molinero, O., Ruíz, R., Salguero, A., De la Vega, R. y Márquez, S. (2014). La resiliencia en el deporte: fundamentos teóricos, instrumentos de evaluación y revisión de la literatura. Cuadernos de Psicología del Deporte, 14(3), 83-98. https://doi. org/10.4321/s1578-84232014000300010

Giles, B., Goods, P., Warner, D., Quain, D., Peeling, P., Ducker, K., Dawson, B. y Gucciardi, D. (2018). Mental toughness and behavioural perseverance: A conceptual replication and extension. Journal of Science and Medicine in Sport, 21(6), 640-645. https://doi.org/10.1016/i.jsams.2017.10.036

González, L., Castillo, I. y Balaguer, I. (2019). Exploring the Role of Resilience and Basic Psychological Needs as Antecedents of Enjoyment and Boredom in Female Sports. Revista de Psicodidáctica (English ed.), 24(2), 131-137. https://doi.org/10.1016/j. psicoe.2019.02.001

González, J., Gomariz, M., Valero, A. y Gómez, M. (2020). Resilient Resources in Youth Athletes and Their Relationship with Anxiety in Different Team Sports. International Journal of Environmental Research and Public Health, 17(15), Artículo 5569. https://doi. org/10.3390/ijerph17155569

Isorna, M. y Felpeto, M. (2014). Relación entre la práctica de la actividad física-deportiva y la mejora del bienestar físico-psicológico en personas mayores. Lúdica Pedagógica, 1(19), 11-26. https://doi.org/10.17227/01214128.19/udica11.26

Kegelaers, J. y Wylleman, P. (2019). Exploring the coach's role in fostering resilience in elite athletes. Sport, Exercise, and Performance Psychology, 8(3), 239-254. https://doi.org/10.1037/ spy0000151 
Kilic, Y. y Yildirim, E. (2020). Investigation of Mental Toughness Levels of Individuals Who Actively Do Sports: A Sample of the City of Elazig. Journal of Education and Learning, 9(2), 160. https:/l doi.org/70.5539/jel.v9n2p160

Kristjánsdóttir, H., Jóhannsdóttir, K., Pic, M. y Saavedra, J. (2019). Psychological characteristics in women football players: Skills, mental toughness, and anxiety. Scandinavian Journal of Psychology, 60(6), 609-615. https://doi.org/10.1111/sjop.12571

León-Guereño, P., Tapia, M. y Sánchez, P. (2020). The relationship of recreational runners' motivation and resilience levels to the incidence of injury: A mediation model. PLOS ONE, 15(5), Artículo e0231628. https://doi.org/10.1371/journal.pone.0231628

Madrigal, L., Hamill, S. y Gill, D. L. (2013). Mind over matter: The development of the mental toughness scale (MTS). Sport Psychologist, 27(1), 62-77. https://doi.org/10.1123/tsp.27.1.62

Moen, F., Hrozanova, M., Stiles, T. y Stenseng, F. (2019). Burnout and Perceived Performance Among Junior Athletes-Associations with Affective and Cognitive Components of Stress. Sports, 7(7), 171. https://doi.org/10.3390/sports 7070171

Moher, D., Liberati, A., Tetzlaff, J., Altman, D. G. y Prisma Group. (2009). Preferred reporting items for systematic reviews and meta-analyses: the PRISMA statement. PLoS Medicine, 6(7), Artículo e1000097. https://doi.org/10.1371/journal.pmed. 1000097

Moo, J. y Solís, O. (2019). Resiliencia y lesiones en deportistas universitarios. Revista de Psicología de la Salud, 7(1), 134-151. https://doi.org/10.21134/pssa.v7i1.867

Moreno, B., Muñoz, M., Cuellar, J., Domancic, S. y Villanueva, J. (2018). Revisiones Sistemáticas: definición y nociones básicas. Revista clínica de periodoncia, implantología y rehabilitación oral, 17(3), 184-186. https://doi.org/10.4067/s071901072018000300184

Oliveira, A. (2014). Estudo das Propriedades Psicométricas da Escala de Resiliência, versão breve (RS13-A): A resiliência e a relação com a sintomatologia depressiva numa amostra de adolescentes portugueses. [Tese de Mestrado], Universidade de Coimbra, Faculdade de psicologia e de ciências da Educação.

Onturk, Y., Efek, E. y Yildiz, M. (2020). Investigating the psychological resilience of students in sports sciences faculty. International Journal of Educational Methodology, 6(2), 393-403. https:/l doi.org/10.12973/ijem.6.2.393

Pedro, S. y Veloso, S. (2018). Explorando la resiliencia en deporte. Apoyo del entrenador a la autonomía y compromiso del Atleta - Una contribución a la literatura [Exploring resilience in sports. Coach's autonomy support and athletes engagement -A contribute to literature]. Cuadernos de Psicología del Deporte, 18(1), 151-160. https://doi.org/10.6018/cpd
Ramírez, I., Sánchez, M., Zurita, F., Puertas, P., González, G. y Ubago, J. L. (2020). Multidimensional self-Concept Depending on Levels of resilience and the Motivational Climate Directed towards Sport in Schoolchildren. International Journal of Environmental Research and Public Health, 17(2), 534. https://doi. org/10.3390/ijerph17020534

Reche, C., Martínez, A. y Ortín, F. (2020). Caracterización del deportista resiliente y dependiente del ejercicio físico. Cultura, Ciencia y Deporte, 15(43), 17-26.

Sheard M, Golby J. yVan Wersch A. (2009) Progress toward construct validation of the Sports Mental Toughness Questionnaire (SMTQ). European Journal of Psychological Assessment. 25(3):186-93. https://doi.org/10.1027/7015-5759.25.3.186

Smith, B. W., Dalen, J., Wiggins, K., Tooley, E., Christopher, P. y Bernard, J. (2008). The brief resilience scale: assessing the ability to bounce back. International Journal of Behavioral Medicine, 15(3), 194-200.

Trigueros, R., Aguilar-Parra, J., Álvarez, J., González-Bernal, J. y López-Liria, R. (2019). Emotion, Psychological Well-Being and Their Influence on Resilience. A Study with Semi-Professional Athletes. International Journal of Environmental Research and Public Health, 16(21), Artículo 4192. https://doi.org/10.3390/ ijerph16214192

Trigueros, R., Aguilar-Parra, J., Cangas-Díaz, A., Fernández-Batanero, J., Mañas, M., Arias, V. y López-Liria, R. (2019). The influence of the trainer on the motivation and resilience of sportspeople: A study from the perspective of self-determination theory. PLOS ONE, 14(8), Artículo e0221461. https://doi.org/10.1371/ journal.pone.0221461

Trigueros, R., Álvarez, J. F., Aguilar-Parra, J. M., Alcaraz-Ibáñez, M. y Rosado, A. (2017). Validación y adaptación española de la escala de resiliencia en el contexto deportivo (ERCD). Psychology, Society \& Education. 9(2), 311-324.

Trigueros, R., Mercader, I., González-Bernal, J., Aguilar-Parra, J., González, J., Navarro, N. y Soto, R. (2020). The Influence of the Trainer's Social Behaviors on the Resilience, Anxiety, Stress, Depression and Eating Habits of Athletes. Nutrients, 12(8), Artículo 2405. https://doi.org/70.3390/nu12082405

Vigário, l., Serpa, S. y Rosado, A. (2009). Tradução e Adaptação da Escala de Resiliência para a população portuguesa. Universidade Técnica de Lisboa, Facultade de Motricidad Humana.

Wagnild, G.M. y Young, H. M. (1993). Development and psychometric evaluation of the Resilience Scale. Journal of Nursing Measurement. 1(2). 165-177. https://doi.org/10.1016/j.psychsport.2018.09.006 